\title{
Cluster Based Hybrid Routing Mechanism for Mobility Management in Wireless Mesh Networks
}

\author{
D. Usharani, P.Venkata Krishna
}

\begin{abstract}
Wireless mesh networks are the new advancement. In addition, they are enticing large number of researchers with their continuous utilization of the internet. However, providing QoS guarantee to the mobility of the nodes in the network is a thought-provoking problem. To overcome this, a hybrid routing mechanism for QoS management in wireless mesh networks is proposed. As an initial step, the clustering formation is developed for managing the scalability issues in mesh network. The Inter mesh routing and Intra mesh routing is developed for QoS guarantee routing for data forwarding in mesh networks. The evaluation of the proposed protocol is analysed under different parameters. The consequences of simulation show that the performance of proposed protocol is good in different conditions.
\end{abstract}

Keywords: Cluster head, Inter mesh routing, intra mesh routing, clustering.

\section{INTRODUCTION}

Wireless mesh networks (WMNs) [1] is treated as one of the promising technology in the field of computer networks. WMNs provide better network connectivity for the mobile nodes that are participating in the communication. WMNs have the major advantages of robustness, easy deployment and cost effectiveness. In general, WMNs are composed of three nodes such as member nodes, routers and gateways. The member nodes are the clients for the WMNs, routers is responsible for transmitting the packets form one to another node and the gateways are the devices which connected to the internet. The functionality of the gateway is designed with two types of data traffics; intranet and internet. The intranet creates communication between the two nodes that are of same wireless mesh network. The internet forwards packet through the gateway. This gateway passes the packets to the nodes.

The nodes in the wireless mesh networks will have the capability of establishing the connection with other nodes in the network on their own. This type of practice leads the benefit of WSN in terms of reliability, low installation cost, self-management and large scale deployment. The foremost benefits of WMNs are wide area of network, little installation budget and high fault tolerance. The general structure of WMNs is given in figure 1 .

Revised Manuscript Received on 14 August, 2019.

D. Usharani, Department of Computer Science, Sri PadmavatiMahilaVisvavidyalayam, Tirupati, AndhraPradesh, India

P.Venkata Krishna, Department of Computer Science, Sri PadmavatiMahilaVisvavidyalayam, Tirupati, AndhraPradesh, India(Email: pvk@spmvv.ac.in)

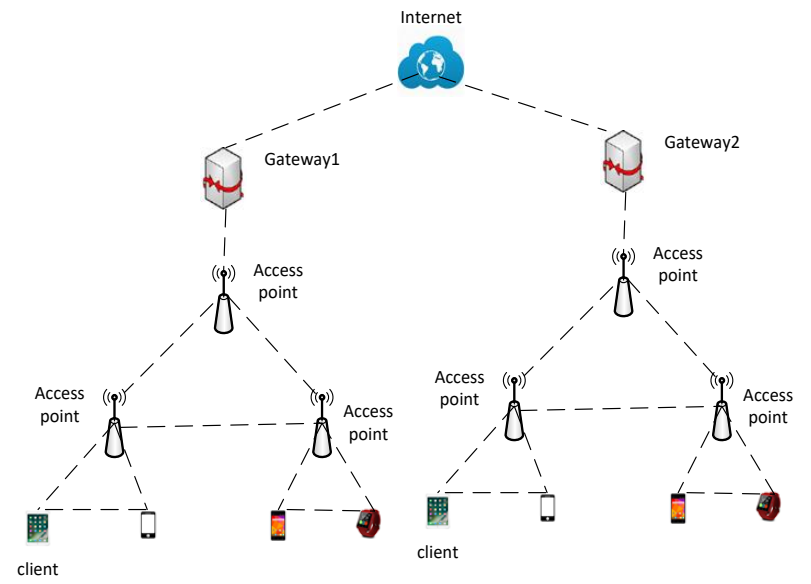

Figure 1: Sample Wireless Mesh Network Architecture

The major challenge faced by the WMNs are providing the network connectivity at the time of node mobility form one router to the another router. To resolve this problem, many investigators contributed diverse strategies for movement administration such as mesh movement administration [2], wireless mesh mobility management [3] and mobility management [4]. Mesh mobility management follows the forward chain model for controlling the packets. This model is efficient in reducing the control overhead, but the session rate is high and it impacts in packet delivery ratio. Wireless mesh mobility management is introduced to control the overhead and also to decrease the size the forward chain and mobility management model does not contain the mechanisms for controlling the packet overhead. The major problem of all the stated management models is that they are following the same properties for all the nodes. Therefore, a mobility model should consider the individual properties of the nodes for mobility management.

In this paper, a hybrid routing protocol was proposed for managing the mobility in the WMNs. The hybrid routing protocol address the cluster formation, inter and intra mesh routing protocol. The proposed protocol considers the QoS parameters for selecting the route between the sending node to receiving node.

The remaining paper is structured as follows. Section 2 presents about the literature survey regarding the routing protocols in WMNs. Section 3 deals with the hybrid routing protocol for WMNs. Section 4 explains about the experimental valuation with respect to overhead of the path 
and throughput. Finally the conclusion is presented in section 5 .

\section{II.RELATED WORK}

To address the movement administration in wireless mesh nets, many approaches are proposed. The handoff strategy which is depending on position administration is proposed by Ganguly et al. in [4]. The performance evaluation of the proposed mechanism shows that the latency of the tunnelling routing is much high when compared to the flat routing. In [5], SMesh was proposed for handling the multiple access points in the WMNs. This mechanism has the ability to reduce the latency in the handoff mechanism. Generally, movement administration is categorised in to two kinds; one strategy is grounded on routing and the other on tunnelling. The prior strategy is developed for IP based networks. Cellular IP [6], HAWAII [7], iMesh [8] are the basic mobility management schemes proposed for wireless mesh networks. The integration of mobility management with the routing protocols makes the network to identify the location of the nodes. Another type of routing mechanism in mobility management is tunnelling based mechanisms. Some of the examples for tunnelling based mechanisms are MIP-RR [11], Mobile IP [13], IDMP [14], Ant [15] and Hierarchical Mobile IPv6 [12].

In routing based mechanisms, the routers maintain the routing tables along with location details of the mobile nodes where the packets to be routed. In cellular network [6], mobile nodes utilizes the gateway IP address as the location address and every router in the WMN utilize the location address of the nodes to upstream or to downstream the packets. In HAWAII [7], each mobile node has their own IP address. These IP addresses are allocated by the gateways and router uses the IP address of the mobile node for upstream and downstream of the packets. iMesh[8] routing protocol works with the principles of AODV [9] and OLSR [10] of mobile ad hoc networks. These protocols are dependent on the traffic broadcasting, route discovery and location identification. These types of mechanisms will increase the routing overhead in mobile nodes.

In tunnelling based mechanisms, a central server is maintained to update the information of mobile nodes along with their location information such as home agents in Mobile IP [13] and foreign agents in MIP-RR [11], it incurs signalling cost for sending the location update messages. This is a critical problem in routing by sending the update messages for every location change. In Ant [15], the mobile node needs to send the location update information for central server, if the nodes change the router. This creates the routing overhead to the wireless mesh network.

\section{PROPOSED ROUTING PROTOCOL FOR WIRELESS MESH NETWORK}

In the proposed hybrid routing protocol, the combination of Inter mesh routing protocol (IMRP) and Intra mesh routing protocol (IRP) are used for communications. The IMRP is used to develop the communication with in the infrastructure and IRP is used to create the communication to the external network, i.e., Internet. However, in order to improve the performance of the proposed protocol, clustering mechanism is used to divide the network in to number of sub groups called as clusters. Each cluster is organized by the cluster head. This clustering mechanism will optimize the load of the network by limiting the control messages to the cluster groups. This type of practice will helps in minimizing the dimensions of the routing table and improves the route finding procedure.

The proposed routing mechanism deals with three modules; cluster elaboration, IMRP and IRP. Before that the proposed mechanism needs to define the methods for identifying the neighbouring node information by considering the QoS metrics.

\subsection{Managing the Neighbouring Node}

The data of the Neighbouring node is very critical for the proposed protocol to provide the network topology, to form the clusters and to maintain the QoS for routing. Hello packet is transmitted intermittently by every node to order to obtain data about the neighbouring node. Figure 2 shows the format of the Hello packet. The network nodes will bring their neighbour table up-to-date with the IP address, Cluster head IP address, weight, QoS metric when they receive the Hello packet. The QoS parameter includes delay, available bandwidth and Jitter. The Neighbouring table is given in Figure 3.

\begin{tabular}{|c|c|c|c|}
\hline Node IP & Cluster head IP & Weight & $\begin{array}{c}\text { Utilized } \\
\text { bandwidth }\end{array}$ \\
\hline
\end{tabular}

Figure 2: Hello Packet Format

\begin{tabular}{|c|c|c|c|}
\hline Node IP & Cluster head IP & Weight & $\begin{array}{c}\text { Q.0S } \\
\text { Parameter }\end{array}$ \\
\hline
\end{tabular}

Figure 3: Format of Neighbouring Table

\subsection{Factors of Quality of Service}

The major aim of the proposed hybrid routing protocol is to find the links based on the QoS parameters. Delay, available bandwidth and jitter are the set of QoS parameters used in the proposed protocol.

Delay: The quality parameter delay is measured based on the Hello messages round rip time, i.e, the time taken from initiation of the hello message and to receive the response.

Available bandwidth: To calculate the available bandwidth of the network, utilized bandwidth of each node is considered along with the neighbouring nodes expressed in hello message.

$$
B W(x)=B W_{T}-\sum_{x=N(x)} B_{\text {utilized }}(x)
$$

Where $\mathrm{BW}(\mathrm{x})$ denotes the bandwidth that is available for the node $\mathrm{x}, \mathrm{BW}_{\mathrm{T}}$ denotes the whole bandwidth allocated, $\mathrm{B}_{\text {utilized }}(\mathrm{x})$ denotes the utilized bandwidth of $\mathrm{x}$ and their neighbouring nodes.

Jitter: This QoS metric is defined by calculating the mean value for the variation of recorded trip time of hello messages for a particular time.

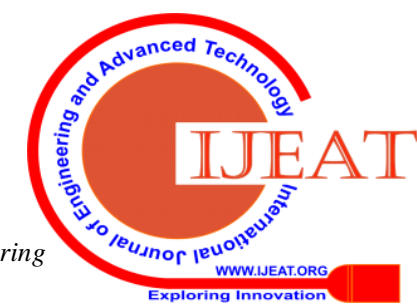




\subsection{Cluster Management in WMNs}

The proposed cluster management procedure is a combination of both LID grounded clustering procedure [16] and Weight grounded clustering procedure [17]. The election of cluster head is carried out by Weight grounded clustering procedure and the election of cluster members are selected based on the nodes having the lowest weight when compared to their neighbouring nodes. This practice is repeated to all the nodes and every network node is allocated to one of the clusters. Both cluster gateway and distributed gateway is maintained for managing the communication with in the clusters. The proposed algorithm is composed of single hop clusters to minimize the burden in control messages and to observe the connectivity between the gateways and cluster heads. This type of practice is very crucial for deploying the inter process routing. Figure 4 shows the cluster formation in the wireless mesh network

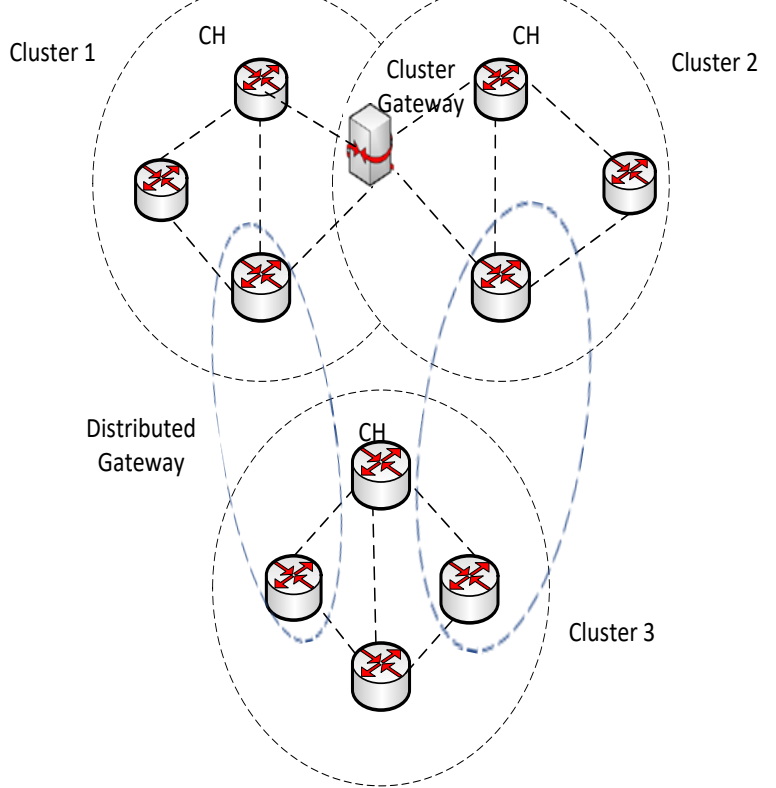

Figure 4: Cluster management in WMN

The cluster management is composed of three different modules: weight module, cluster head selection module and cluster elaboration module. The weight module calculates the weight of each node by adapting the method of weight based clustering algorithm [17]. The node degree and the transmission power are considered for calculating the weight of the node. An additional parameter such as transmission power towards mesh gateway is considered for the node weight. Equation 2 shows the weight factor of the node $\mathrm{x}$.

$$
W(x)=\alpha \times D(x)+\beta \times P(x)+\gamma \times P_{M G}(x)
$$

Here $\mathrm{W}(\mathrm{x})$ represents the node's weight, $\alpha, \beta, \gamma$ are the variables and their sum is equal to $1, \mathrm{D}(\mathrm{x})$ is the node's degree that depends on the total of neighbouring nodes associated with it. $\mathrm{P}(\mathrm{x})$ is the transmission power of the node, $\mathrm{P}_{\mathrm{MG}}(\mathrm{x})$ denotes the transmission power of the node towards mesh gateway.

In the cluster head section module all the nodes are in the initial stage, i.e. weight $=0$ for all the nodes. All the nodes contain the information of neighbouring nodes along with their weights. The nodes weights for $\mathrm{T}$ seconds before the cluster head selection and update their neighbouring table. After $\mathrm{T}$ seconds, the node which having the lowest weight
W is selected as cluster head. The selected cluster head will broadcast hello message to the neighbouring nodes Procedure 1 shows the cluster head selection process.

Procedure 1: Determination of Cluster head
If the weight of Node $\mathrm{x}$ is minimum in the neighbouring table then
Select $\mathrm{x}$ as $\mathrm{CH}$
The IP of node $\mathrm{x}$ is made as IP of cluster head
Transmit Hello Packet $\left\{\mathrm{IP}_{\mathrm{x}}, \mathrm{CH}-\mathrm{IP}_{\mathrm{x}}, \mathrm{W}_{\mathrm{x}}, \mathrm{B}_{\text {utilizad }}\right\}$
End If

The cluster elaboration module is composed of clusters and each cluster is formed based on the hello messages exchanged by the nodes and their associated neighbours. In the cluster elaboration module, the node which receives the hello message form the cluster head $(\mathrm{CH})$ becomes the cluster member (CM). The $\mathrm{CM}$ will act as gateway node when it will interconnect two clusters which are given in figure 2. The CM becomes cluster gateway when it accepts the hello message from the neighbouring cluster $\mathrm{CH}$. The table format for the gateway is given in figure 5 .

\begin{tabular}{|c|c|c|c|}
\hline $\begin{array}{c}\text { Gateway } \\
\text { Type }\end{array}$ & CH1-IP & Ch2-IP & $\begin{array}{c}\text { Distributed } \\
\text { Gateway-IP }\end{array}$ \\
\hline
\end{tabular}

Figure 5: Gateway Table

The gateway information message is sent to the host $\mathrm{CH}$ and neighbour cluster $\mathrm{CH}$. After receiving this message, both $\mathrm{CHs}$ update their neighbouring cluster head table. The format for the gateway information message and neighbouring cluster head table is given in Figures 6 and 7 .

\begin{tabular}{|l|c|c|c|c|}
\hline Gateway IP & Weight & $\begin{array}{c}\text { Gateway } \\
\text { Type }\end{array}$ & CH-IP & $\begin{array}{c}\text { Neighbouring } \\
\text { Cluster CH-IP }\end{array}$ \\
\hline
\end{tabular}

Figure 6: Gateway Information Message format

\begin{tabular}{|c|c|c|}
\hline $\begin{array}{c}\text { Neighbouring } \\
\text { cluster } \mathrm{CH} \text {-IP }\end{array}$ & Gateway-IP & $\begin{array}{c}\text { Gateway } \\
\text { Type }\end{array}$ \\
\hline
\end{tabular}

Figure 7: Neighbouring cluster head table

\subsection{Intra mesh routing protocol (IRP)}

Intra mesh routing protocol is used to find the paths from source to destination to forward the packets. The source node and destination node are located within the same infrastructure. IRP guarantee the QoS by considering the parameters such as delay, bandwidth and jitter for each node at the time of route discovery.

Algorithm 2 explains about the route discovery process in intra mesh routing protocol. A source node $\mathrm{A}$ is initiated to forward the packets to the destination B by verifying the QoS parameters or by considering the entry of node B in the routing table. If the terminus node $\mathrm{B}$ is not found, then the protocol executes the route discovery process.

Published By:

Blue Eyes Intelligence Engineering

\& Sciences Publication 

NETWORKS

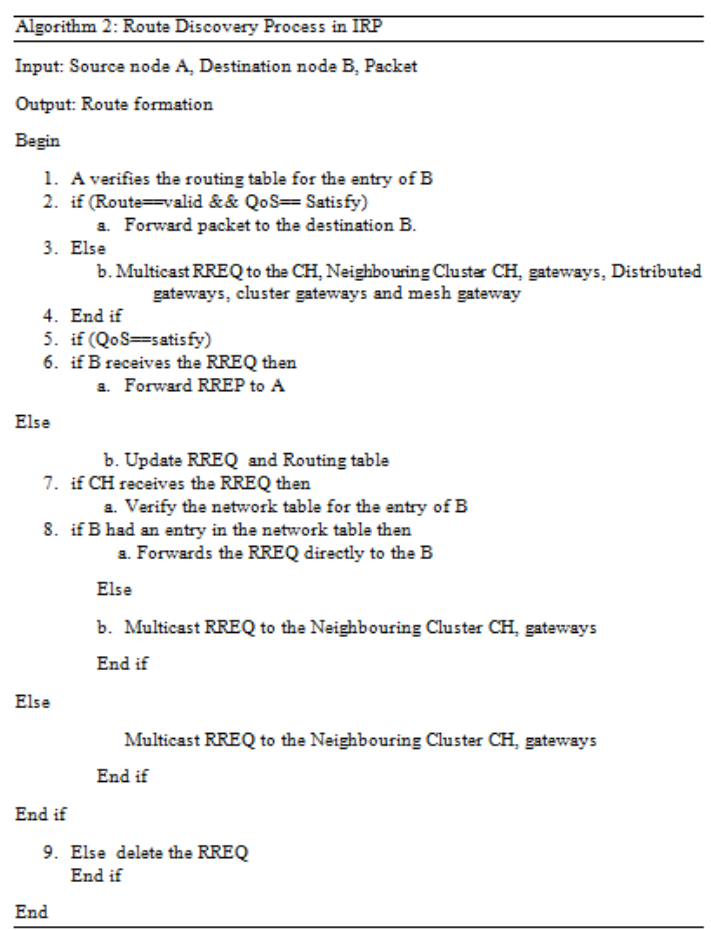

\subsection{Inter Mesh routing protocol (IMRP)}

Inter mesh routing protocol is associated with the communication outside of the infrastructure i.e, the internet. In IMRP all the traffic is transferred through the mesh gateway to communicate with external networks, so we adopt the tree based routing mechanism for communication. The mesh gateway is act as root node and the remaining nodes are act as child nodes. Cluster members are not considered for tree formation. Algorithm 3 describes about the route selection process in IMRP. The route selection process considers three paths for each node. The first path is evaluated based on the delay and jitter along with satisfied bandwidth. The second path is evaluated based on the delay only and the third path is completely disjoint from other two paths.

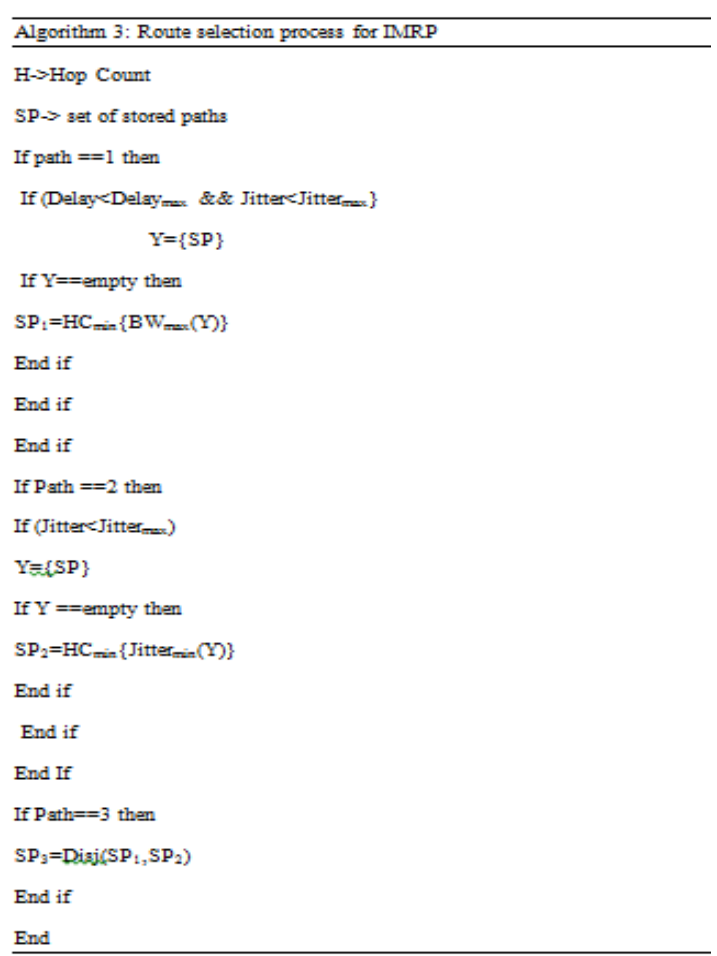

\section{EXPERIMENTAL EVALUATION\& RESULTS}

The behaviour of the projected model is assessed by means of the well-known network simulator called as NS2. The proposed protocol is tested with different scenario in terms of route discovery and overhead of the routing. The simulation scenario is configured with variable number of nodes such as 6 to 25 , packet size is fixed to the 512 bytes, and the traffic is selected as constant bit rate and with a fixed data rate of $512 \mathrm{Kbps}$.

The behaviour of the projected procedure is compared with Distributed Clustering Procedure for Mesh networks (DCA) [18] and QoS based routing algorithm (QoSR) [19]. The routing overhead in the proposed and existing algorithms is posed by RREQ, RREP and Hello packets. Figure 8 demonstrates the RREQ overhead varieties alongside variation in number of nodes. The RREQ overhead of the projected procedure will increment with the quantity of nodes and projected procedure advances the replicated messages to the destination. Therefore, the proposed protocol record highest RREQ overhead compared to the DCA and QoSR.

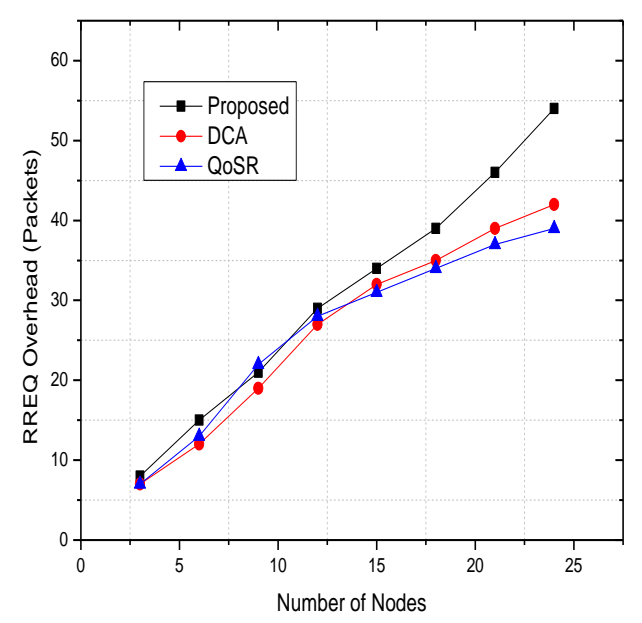

Figure 8: RREQ Overhead Vs Number of Nodes

Figure 9 and Figure 10 demonstrates that projected procedure has a smaller amount RREP and Hello packet overhead. This is because the DCA and QoSRenables the transitional nodes to transmit the RREP packets. In projected procedure, just the receiving node will transmit the RREP packet. Figure 10 demonstrates that the Hello packets transmitted by the nodes in the projected procedure are a smaller amount and it is conversely proportionate to the quantity of RREQ packets transmitted. 


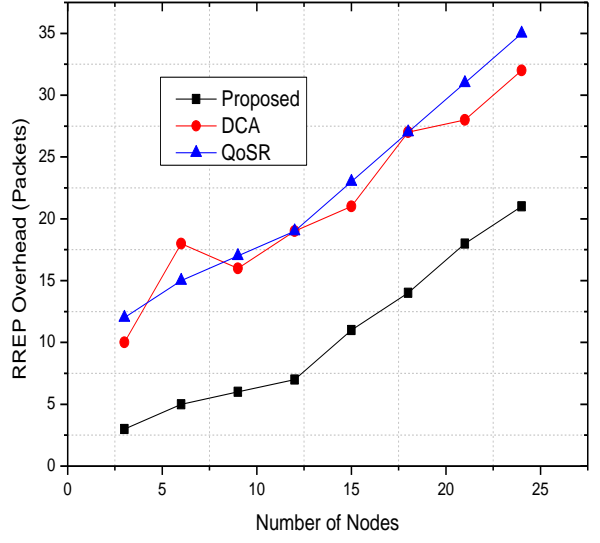

Figure 9: RREP Overhead Vs Number of Nodes

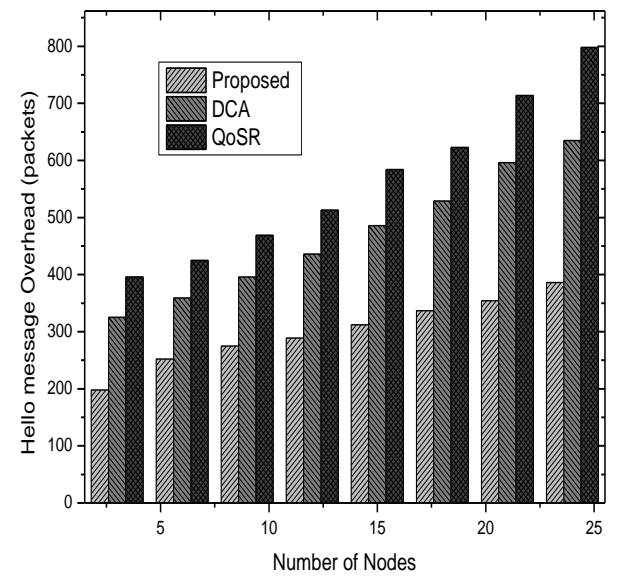

Figure 10: Hello Message Overhead Vs Number of Nodes

Figure 11 demonstrates the time utilization of path finding procedure dependent on the system dimensions. The time utilization of the three conventions for path finding procedure increments regarding the quantity of nodes. Be that as it may, the projected procedure invested few time in discovering the path when contrasted with the rest of the procedures. The projected procedures chooses the better path dependent on the QoS parameters.

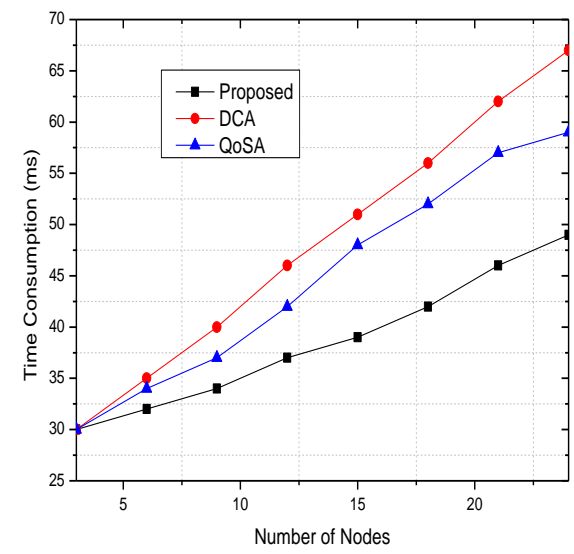

Figure 11: Time Consumption for Route Discovery
Figure 12 demonstrates the outcomes with respect to throughput. Here, it very well may be seen that the projected procedure has the preferable throughput over the rest of the procedures with variation dimensions of the network. The projected procedures decreases the interediary nodes and chooses the nodes dependent on the QoS parameters. Subsequently, the throughput is enhanced for the projected procedure.

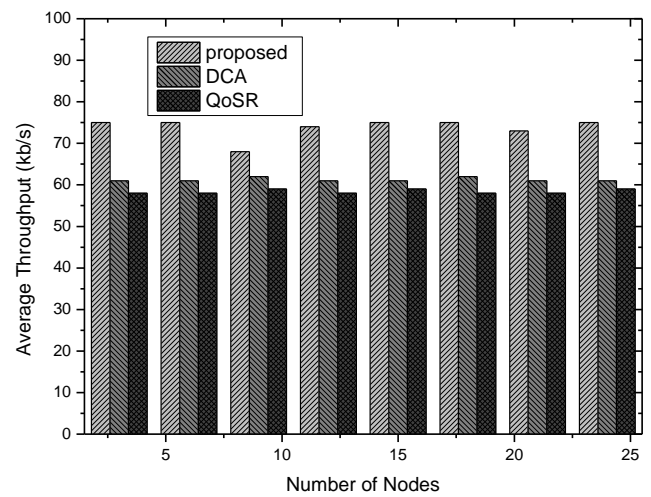

Figure 12: Average Throughput of Proposed, DCA and QoSR

\section{CONCLUSION}

Hybrid routing protocol for QoS guarantee routing in wireless mesh networks is proposed in this paper. The hybrid routing protocol is the combination of Inter mesh routing protocol (IMRP) and Intra mesh routing protocol (IRP). The election of cluster head is carried out by Weight grounded clustering procedure and the election of cluster members are selected based on the nodes having the lowest weight when compared to their neighbouring nodes. The QoS factors deliberated in this paper are delay, available bandwidth and jitter. The behaviour of the projected protocol is tested with time ingestion of path finding, routing overhead and ordinary throughput. The projected protocol proved their efficiency when equalled to the DCA and QoSR protocols.

\section{REFERENCES}

1. R. Huang, C. Zhang, and Y. Fang, "A mobility management scheme for wireless mesh networks," in Proceedings of the $50^{\text {th }}$ Annual IEEE Global Telecommunications Conference (GLOBECOM'07), pp. 5092-5096, November 2007.

2. D. Huang, P. Lin, and C. Gan, "Design and performance study for a mobility management mechanism.(WMM) using location cache for wireless mesh networks," IEEE Transactions on MobileComputing, vol. 7, no. 5, pp. 546-556, 2008.

3. M. Ren, L. Chao, Z.Huizhou, Z. Tong, and Y.Wei, "MEMO: an applied wireless mesh network with client support and mobility management," in Proceedings of the 50th Annual IEEE GlobalTelecommunications Conference (GLOBECOM'07), pp. 164-170, November 2007.

4. S.Ganguly, V. Navda, K. Kim, A. Kashyap, D.

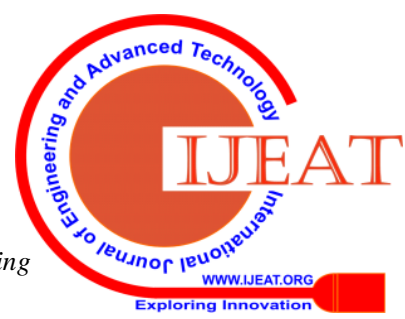


Niculescu, R. Izmailov, S. Hong, and S. Das. Performance optimizations for deploying voip services in mesh networks. IEEE Journal on Selected Areas in Communications, 24(11):2147-2158, November 2006.

5. Y. Amir, C. Danilov, M. Hilsdale, R. Musaloiu-Elefteri, and N. Rivera. Performance of ip micro-mobility management schemes using host based routing. In Proceedings of the 4th international conferenceon Mobile systems, applications and services (MobiSys'06), Uppsala, Sweden, June 2006.

6. A. Campbell et al., "Design implementation and evaluation of cellular IP", IEEE Personal Commun., vol. 7, no. 4, pp. 42-49, 2000.

7. R. Ramjee et al., "HAWAII: a domain-based approach for supporting mobility in wide-area wireless networks", Proc. 1999 Int. Conf on Network Protocols, pp. 283-292.

8. V. Navda, A. Kashyap, S. Das, "Design and evaluation of imesh: an infrastructure-mode wireless mesh network", Proc. 2005 IEEE Int. Symposium on a World of Wireless Mobile and Multimedia Networks, pp. 164170.

9. Zhang, R. W. Pazzi, A. Boukerche, "A mobility management scheme for wireless mesh networks based on a hybrid routing protocol", Computer Networks, vol. 54, no. 4, pp. 558-572, Mar. 2010.

10. P. Jacquet et al., "Optimized link state routing protocol for ad hoc networks", Proc. 2001 IEEE INMIC, pp. 6268.

11. E. Gustafsson, A. Jonsson, and C. Perkins. Mobile ip regional registration. Internet Draft, 2000.

12. R. Hsieh, A. Seneviratne, H. Soliman, and K. El-Malki. Performance analysis on hierarchical mobile ipv6 with fast-handoff over end-to-end tcp. In Globecom'02, Taipei, R.O.C., November 2002.

13. D. Johnson, C. Perkins, J. Arkko, "Mobility support in IPv6", June 2004

14. A. Misra et al., "IDMP-based fast handoffs and paging in IP-based 4G mobile networks", IEEE Commun. Mag., vol. 40, no. 3, pp. 138-145, Mar. 2002.

15. H. Wang et al., "A Network-based local mobility management scheme for wireless mesh networks", Proc. 2007 IEEE Wireless Communications and Networking Conference, pp. 3792-3797.

16. Lin, Chunhung Richard, and Mario Gerla. "Adaptive clustering for mobile wireless networks." IEEE Journal on Selected areas in Communications 15.7 (1997): 1265 1275.

17. Chatterjee, M., Das, S. K., \&Turgut, D. (2002). WCA: A weighted clustering algorithm for mobile ad hoc networks. Cluster computing, 5(2), 193-204.

18. Langar, R., Bouabdallah, N., \&Boutaba, R. (2009). Mobility-aware clustering algorithms with interference constraints in wireless mesh networks. Computer Networks, 53(1), 25-44.

19. Aoun, Bassam, RaoufBoutaba, Youssef Iraqi, and Gary Kenward. "Gateway placement optimization in wireless mesh networks with QoS constraints." IEEE Journal on Selected Areas in Communications 24, no. 11 (2006): 2127-2136. 